\section{Pressure and flow properties of cannulae for extracorporeal membrane oxygenation II: drainage (venous) cannulae} sagepub.com/journals-permissions DOI: 10.1177/02676591198305/4 journals.sagepub.com/home/prf

(S)AGE

\author{
Lars Mikael Broman, 1,2,3 (iD Lisa Prahl Wittberg, ${ }^{4}$ \\ C Jerker Westlund,' Martijn Gilbers, ${ }^{5,6}$ Luisa Perry da Câmara, ${ }^{7}$ \\ Jan Westin, ${ }^{8}$ Fabio Silvio Taccone, ${ }^{3,9}$ \\ Maximilian Valentin Malfertheiner, 3,10 \\ Matteo Di Nardo, 3,11 (iD Justyna Swol,3,12 \\ Leen Vercaemst, 3,13 Nicholas A Barrett, 3,14 \\ Federico Pappalardo, 3,15 Jan Belohlavek, 3,16 Thomas Müller, 3,10 \\ Mirko Belliato ${ }^{3,17}$ and Roberto Lorusso ${ }^{3,4}$
}

\begin{abstract}
The use of extracorporeal life support devices such as extracorporeal membrane oxygenation in adults requires cannulation of the patient's vessels with comparatively large diameter cannulae to allow circulation of large volumes of blood $(>5 \mathrm{~L} / \mathrm{min})$. The cannula diameter and length are the major determinants for extracorporeal membrane oxygenation flow. Manufacturing companies present pressure-flow charts for the cannulae; however, these tests are performed with water. Aims of this study were I. to investigate the specified pressure-flow charts obtained when using human blood as the circulating medium and 2. to support extracorporeal membrane oxygenation providers with pressure-flow data for correct choice of the cannula to reach an optimal flow with optimal hydrodynamic performance. Eighteen extracorporeal membrane oxygenation drainage cannulae, donated by the manufacturers $(n=6)$, were studied in a centrifugal pump driven mock loop. Pressure-flow properties and cannula features were described. The results showed that when blood with a hematocrit of $27 \%$ was used, the drainage pressure was consistently higher for a given flow (range 10\%-350\%) than when water was used (data from each respective manufacturer's product information). It
\end{abstract}

\footnotetext{
'ECMO Centre Karolinska, Department of Pediatric Perioperative Medicine and Intensive Care, Karolinska University Hospital, Stockholm, Sweden

2Department of Physiology and Pharmacology, Karolinska Institutet, Stockholm, Sweden

${ }^{3}$ Working Group on Innovation and Technology, EuroELSO, Newcastle upon Tyne, UK

${ }^{4}$ The Linné Flow Centre \& BioMEx, Department of Mechanics, KTH Royal Institute of Technology, Stockholm, Sweden

${ }^{5}$ Department of Cardio-Thoracic Surgery, Heart \& Vascular Centre, Cardiovascular Research Institute Maastricht (CARIM), Maastricht University Medical Hospital, Maastricht, The Netherlands ${ }^{6}$ Department of Physiology, Maastricht University, Maastricht, The Netherlands

${ }^{7}$ Centro Hospitalar de Lisboa Central, Hospital Curry Cabral, Lisbon, Portugal

${ }^{8}$ Department of Medical Technology, Karolinska University Hospital, Stockholm, Sweden

${ }^{9}$ Department of Intensive Care, Hôpital Erasme, Université Libre de Bruxelles (ULB), Brussels, Belgium

${ }^{10}$ Department of Internal Medicine II, Cardiology and Pneumology, University Medical Center Regensburg, Regensburg, Germany
}

\footnotetext{
I'Pediatric Intensive Care Unit, Children's Hospital Bambino Gesù, IRCCS, Rome, Italy

${ }^{12}$ Department of Pulmonology, Intensive Care Medicine, Paracelsus Medical University, Nuremberg, Germany

${ }^{13}$ Department of Perfusion, University Hospital Gasthuisberg, Louven, Belgium

${ }^{14}$ Department of Critical Care and Severe Respiratory Failure Service, Guy's and St. Thomas' NHS Foundation Trust, London, UK

${ }^{15}$ Advanced Heart Failure and Mechanical Circulatory Support Program, Vita Salute University, San Raffaele Hospital, Milan, Italy

${ }^{16} 2$ nd Department of Medicine-Department of Cardiovascular Medicine, General University Hospital in Prague and First Faculty of Medicine, Charles University in Prague, Prague, Czech Republic 17U.O.C. Anestesia e Rianimazione I, Fondazione IRCCS Policlinico San Matteo, Pavia, Italy
}

\section{Corresponding author:}

Lars Mikael Broman, ECMO Centre Karolinska, Department of Pediatric Perioperative Medicine and Intensive Care, Karolinska University Hospital, 17176 Stockholm, Sweden.

Email: lars.broman@sll.se 
is concluded that the information provided by manufacturers in line with regulatory guidelines does not correspond to clinical performance and therefore may not provide the best guidance for clinicians.

\section{Keywords}

drainage; cannula; pressure; flow; extracorporeal membrane oxygenation; extracorporeal life support; venous

\section{Introduction}

Vascular access is crucial to perform extracorporeal membrane oxygenation (ECMO). ${ }^{1}$ However, inappropriate choice of vascular access may also expose the patient for potential life-threatening complications. ${ }^{2}$ Following the $\mathrm{H}_{1} \mathrm{~N}_{1}$ pandemic in 2009-2010, adult ECMO cases have increased, with development of mobile ECMO teams and percutaneous approaches to cannulation. ${ }^{2}$ There has also been renewed interest in cannula design and new cannulae entering the market. ${ }^{3,4}$

The drainage cannula is the most important component in determining the flow through the ECMO circuit. There are a multitude of drainage cannula designs and sizes. The characteristics and performance of these cannulae have not been evaluated in an independent investigation. Most manufacturers or researchers present pressure-flow charts using water or glycerol for testing, in line with current recommendations. ${ }^{5}$ These charts, however, may be inaccurate since both water and glycerol solutions are Newtonian fluids, whereas blood is a non-Newtonian, pseudoplastic fluid. ${ }^{6,7}$ The nonNewtonian behavior of blood is characterized by shear thinning behavior, that is, viscosity decreases with increasing shear stress. ${ }^{7-9}$ However, for shear rates above $100 \mathrm{~s}^{-1}$, the literature reports blood viscosity to exhibit Newtonian behavior. ${ }^{10-12}$

Poiseuille's law describes the factors affecting the flow of a Newtonian fluid through a cylindrical tube of constant radius for a laminar flow. In accordance with Poiseuille's law (equation (1)), the radius is the most important factor determining flow, although length, viscosity, and the pressure gradient are key factors as well

$$
\mathrm{Q}=\frac{\mathrm{r}^{4} \cdot \pi \cdot\left(\mathrm{P}_{1}-\mathrm{P}_{2}\right)}{8 \cdot \mathrm{L} \cdot \eta}
$$

In this equation, " $r$ " represents the radius; "(P1-P2)" the pressure-drop, $\Delta \mathrm{P}$; " $\mathrm{L}$ " the length of the cylinder; and " $\eta$ " is the dynamic viscosity.

The flow increases linearly with the pressure gradient across the tube while the flow is laminar; once the flow becomes turbulent, this relationship no longer holds true. ${ }^{8}$ Given that flow is directly proportional by the fourth power of the radius, larger diameter cannulae are required to achieve the high blood flows required for ECMO (often in excess of $5 \mathrm{~L} / \mathrm{min}$ in adults).
Based on the paucity of information regarding bloodbased test for ECMO cannulae, the aim of this study was to present pressure-blood flow charts for peripheral drainage cannulae currently used for ECMO.

\section{Material and methods}

Eighteen cannulae marketed for ECMO (Extracorporeal Life Support Organization (ELSO) Registry, Ann Arbor, MI, USA https://www.elso.org/Registry/Support Documents/CannulaEquipmentList.aspx), were evaluated in this ex-vivo study. Manufacturers supporting the study were offered the research protocol and mock loop blue print for information before agreeing to supply cannulae free of charge to the researchers. This independent investigation did not ask for and would not have accepted any additional support. Test specimens were coded and blinded to investigators. After the measurements had been carried out, each cannula's identification was revealed and double checked against Code key and the result transferred to an Excel (Microsoft, Redmond, WA, USA) spread sheet.

Eighteen cannulae of five different basic designs of various diameters and lengths were tested. Drainage cannulae for adult peripheral cannulation have a greater variety of design features as compared to return cannulae. One unique cannula design was the stent based Smart draining device, a blunt cannula with a vascular stent extending up to the tip. The most common tip designs are the single-staged or Lighthouse alone or in combination with multiple hole sequences further down the stem (staged interrupted) or multi-staged with multiples holes at regular intervals proximally from the tip (Figure 1). All tested cannulae were suitable for percutaneous cannulation. The cannula size is given in French $(\mathrm{Fr})$ which refers to the outer diameter and is converted to millimeters by dividing Fr by 3 $(1 \mathrm{Fr}=1 / 3 \mathrm{~mm})$. In most cases, cannula length corresponds to the maximum insertion length (Figure 1), that is, the distance from the tip to where the cannula starts to taper out (increase in diameter). However, for example, Medtronic (Medtronic Europe Sárl, Tolochenaz, Switzerland) refers the length of its BioMedicus NextGen cannulae as the total length from the proximal $3 / 8^{\prime \prime}$ connector to the tip. Table 1 shows cannula features in detail obtained from manual caliper measurements performed by the researchers. 


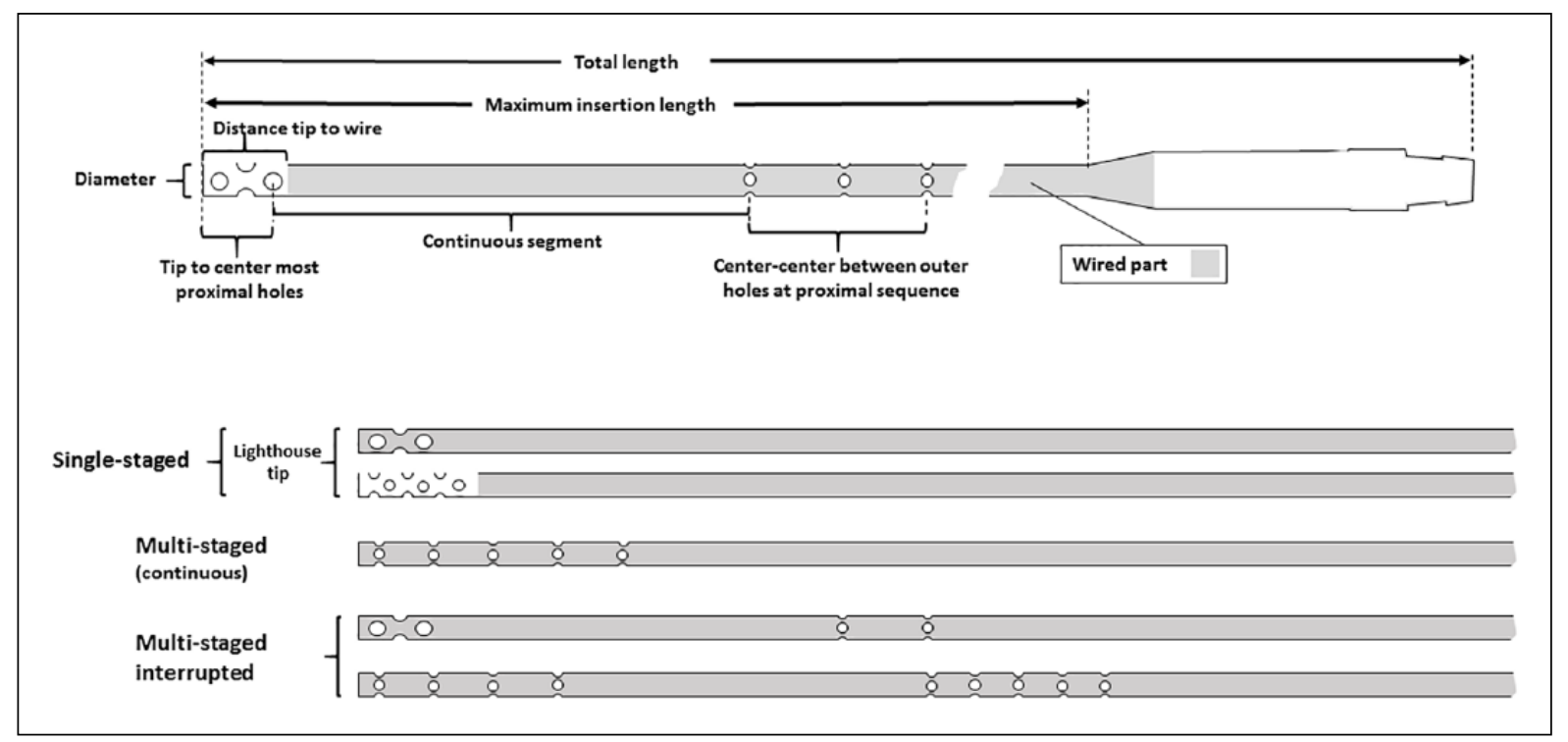

Figure I. The defined measuring points of the drainage cannulae used for extracorporeal membrane oxygenation (ECMO) presented in Table I, and the contemporary tip designs. Multi-stage cannulae usually have four holes per row.

The mock loop was composed of a Thoratec/ Levitronix centrifugal pump (Abbott, Illinois, USA), a reservoir and $1 / 2 "$ and $3 / 8^{\prime \prime}$ tubing as default (Figure 2). For temperature control during the procedure, a Medos HiLite 7000LT (Xenios, Heilbronn, Germany) membrane lung and a blood warmer (HICO Aquaterm 660, Hirtz \& Co., Cologne, Germany) were used. The temperature was set to $37.5^{\circ} \mathrm{C}$. A test chamber was made from a $750 \mathrm{~mm}, 36 \mathrm{~mm}$ inner diameter Plexiglass ${ }^{\mathrm{Tm}}$ tube with Luer-lock ports every $71 \mathrm{~mm}$. Pressures were measured via these ports at the tip and at draining ports along the test cannula body (Figure 2). The cannula was advanced to a point where the most proximal holes were $50 \mathrm{~mm}$ inside of the test chamber.

The cannulae tests were performed with expired human whole blood (HWB) acquired from the hospital's blood bank. The amount of HWB available for the investigations was $2.4 \mathrm{~L}$. The evening prior to the tests, an ECMO circuit $(1,450 \mathrm{~mL}$ plus the reservoir) was primed with $2.0 \mathrm{~L} 0.9 \%$ saline and $100 \mathrm{~mL} 20 \%$ albumin and run over night to assess for any leakage. The circuit was blood primed the morning after. A hematocrit feasible in ECMO patients of $27 \%$ was maintained during the tests. ${ }^{13,14}$ Air plus 5\% carbon dioxide was used as sweep gas to mimic a "venous" milieu for the blood pumped through the cannula.

For control of hemoglobin concentration $(\mathrm{Hb})$ and hematocrit (Hct), blood samples were analyzed with an iSTAT device (Abbott Point of Care, Maidenhead, UK). No anticoagulative regimen was used. Blood flow and revolutions per minute (RPM) were controlled via the Thoratec. Flow was independently measured by an ELSA (Transonic Systems Inc., Ithaca, NY, USA) stand-alone unit for higher flow resolution and accuracy (uses two flow probes). Pressures were measured and displayed via the Thoratec.

\section{Pressure measurements and calculations}

The pressure gradient over the cannula for each flow was assessed by measuring the proximal pressure $\left(\mathrm{P}_{2}\right)$ at the Luer-lock port available on the $3 / 8^{\prime \prime}$ cannula connector or such connector adjacent to the cannula adapter. The distal pressure $\left(\mathrm{P}_{1}\right)$ was measured via one Luer-lock port on the test chamber close to the draining port/s (Figure 2). For multi-stage design cannulae, the $\mathrm{P}_{1}$ used for assessment was measured from the base of the most proximal hole sequence, that is, the holes where drainage flow starts. ${ }^{14}$ All pressure transducers were placed in level with the horizontal test chamber, calibrated, and checked for drifting regularly.

From the measurements, the following data were taken to chart: cannula code, date/time, blood temperature $\left({ }^{\circ} \mathrm{C}\right)$, blood flow $(\mathrm{L} / \mathrm{min})$, centrifugal pump RPM, $\mathrm{P}_{1}$ and $\mathrm{P}_{2}(\mathrm{~mm} \mathrm{Hg})$. For quality control, blood gases and hematocrit were checked. The pressure gradient over a venous ECMO cannula was for a given flow defined as $\Delta \mathrm{P}=\mathrm{P}_{2}-\mathrm{P}_{1}$. To enable comparison of the pressure-flow graphs obtained from the above assessment using blood with measurements carried out using water, information was gathered from each manufacturer's results publicly available online on their respective webpage.

Ethical concerns: Ethical approval was waived according to Swedish law and contact with the Department of Transfusion Medicine, Karolinska University Hospital, Huddinge, Stockholm, who donated the blood otherwise 


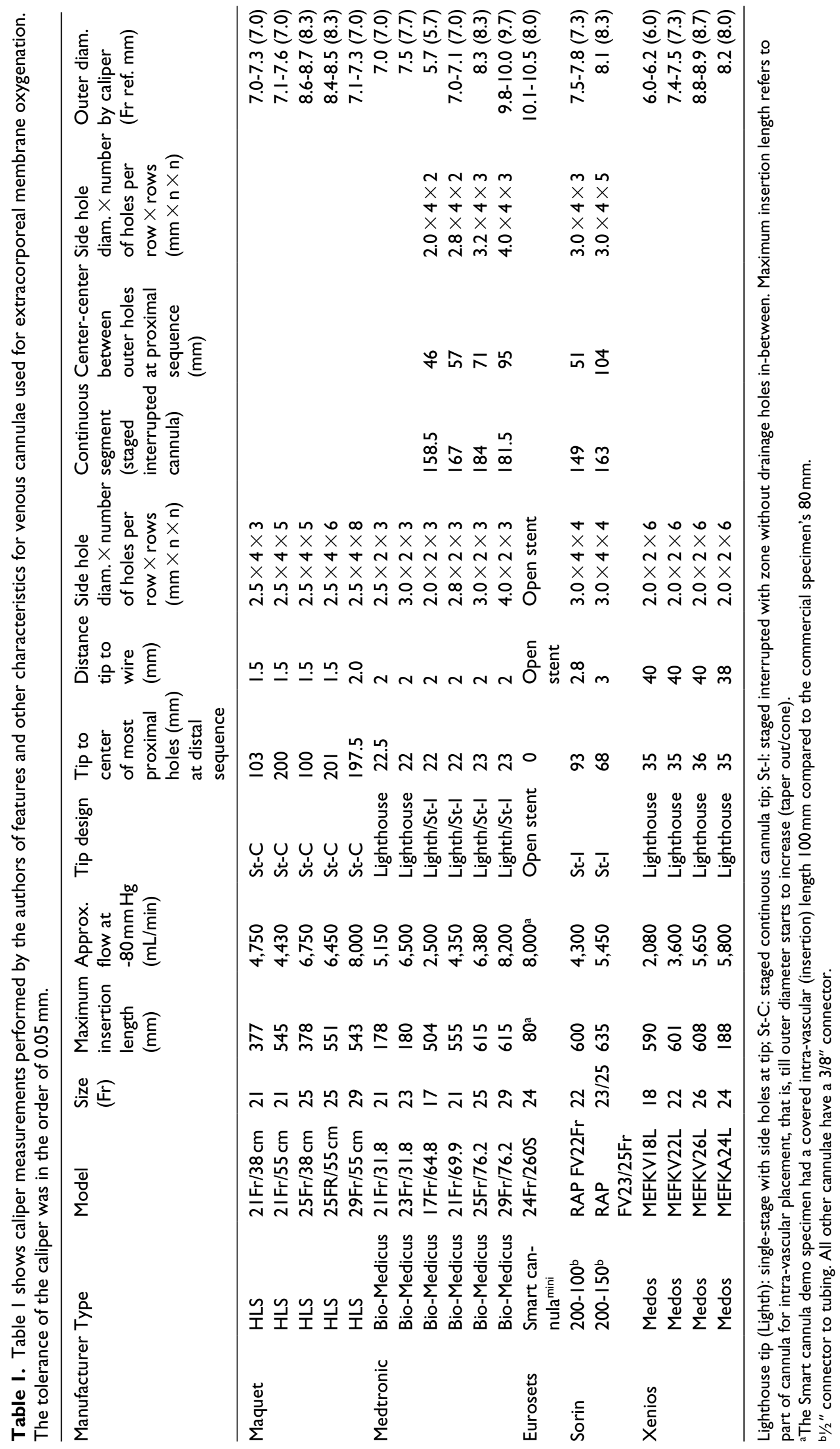




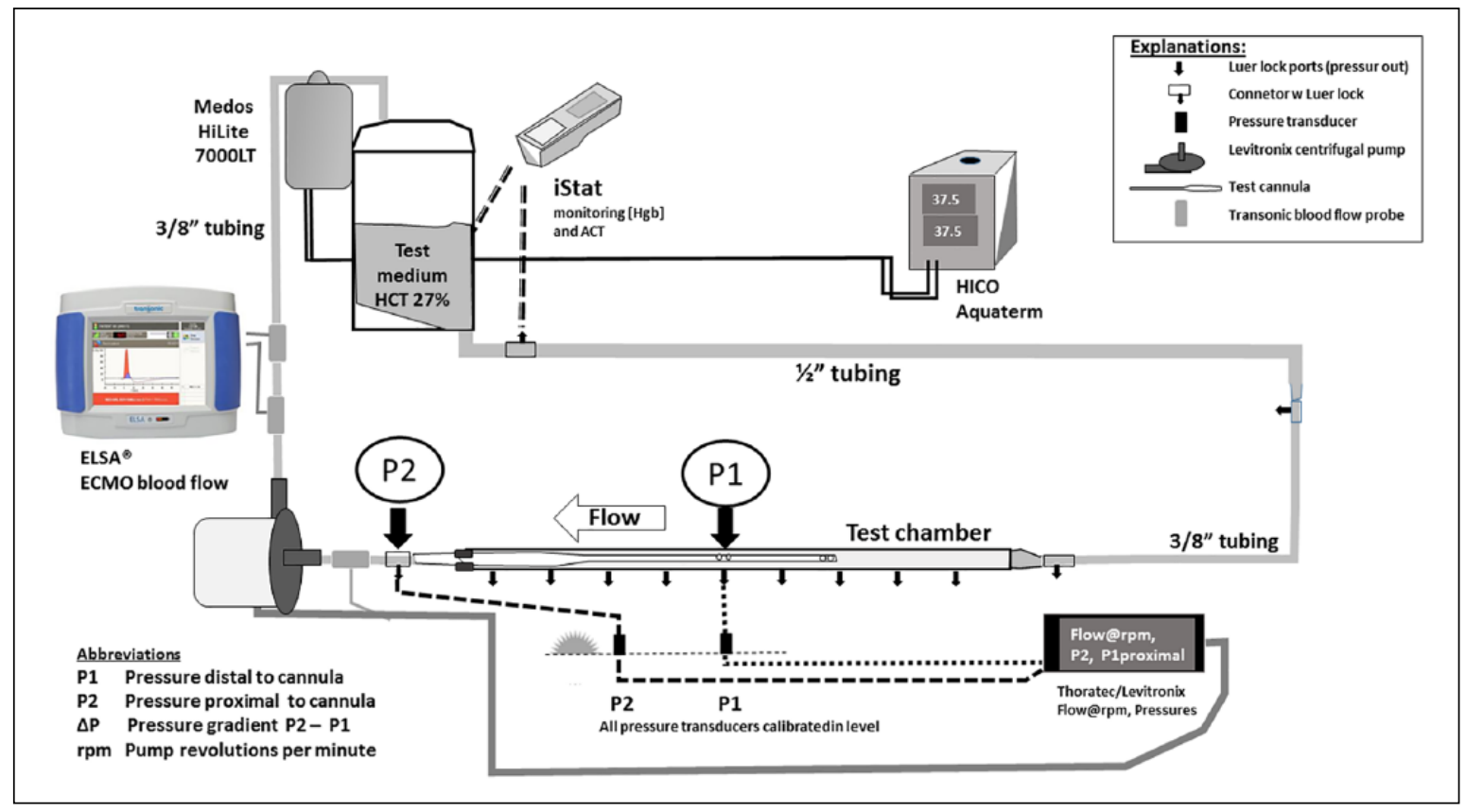

Figure 2. The mock loop for flow tests of draining cannulae used for extracorporeal membrane oxygenation (ECMO).

up for destruction due to passing the 42 days (maximum allowed storage time). Thus, no consent was needed from the donors.

Statistics: Cannulae were not allowed to be directly compared with each other according to agreement with manufacturer. Thus, all results are reported for individual cannula. Consequently, no statistical analysis of the differences between the various cannula flow-pressure relationships was undertaken.

\section{Results}

Eighteen cannulae for venous drainage in ECMO were tested for flow and pressure relationship using whole blood at $37.5^{\circ} \mathrm{C}$. The companies supporting this survey were Eurosets Srl. (Medolla, MO, Italy) with one; Medtronic six; Maquet (Maquet Cardiopulmonary/ Getinge Group, Rastatt, Germany) five, Sorin/Livanova (Milan, Italy) two; and Xenios (Heilbronn, Germany) with four cannulae.

The pressure-flow curves for all tested cannulae are grouped by each manufacturer and presented in Figure 3 . The tests were run to a negative pressure of approximately $-100 \mathrm{~mm} \mathrm{Hg}$. One test was prematurely interrupted due to a communication error. However, based on the sampled data shown for that cannula (Figure 3, Bio-Medicus NextGen $23 \mathrm{Fr} / 31.8 \mathrm{~cm}$ ), the reader may extrapolate the missing part supported by the curves of the neighboring cannulae. When grouping cannulae of same manufacturer and design, the pressure-flow curves are of similar shape, but of different slopes due to variation in cannula size, with the pivoting point at the origin (zero pressure, zero flow).

The pressure-flow relationship varied both between different diameters of the same cannula design and between cannula designs (Figure 4). The curves for an individual family of cannulae were similar. Different brands were not allowed for comparison. However, when pressure-flow curves from tests made with HWB were merged with the manufacturers' water runs, the fractional difference in $\Delta \mathrm{P}$ for flow varied substantially (10\%-350\%) as illustrated in Figure 4 and Supplemental Digital File 1.pdf.

\section{Discussion}

In the current investigation of 18 drainage cannulae for ECMO, five different cannula designs were tested. Performance of each cannula is presented as pressureflow charts pumping HWB. When compared with the flow charts published by the manufacturers (flow data were found online for all cannulae except for the Smart cannula), the $\Delta \mathrm{P}$ needed for a given blood flow was higher when blood was used as compared to water. The additional $\Delta \mathrm{P}$ needed to achieve the same volume flow using HWB compared to water varied for different cannulae. Furthermore, this fractional increase in $\Delta \mathrm{P}$ needed for the same increase in blood flow, decreased with increasing blood flow, an observation supported by the known characterization of blood as a shear thinning fluid. ${ }^{7-9}$ 


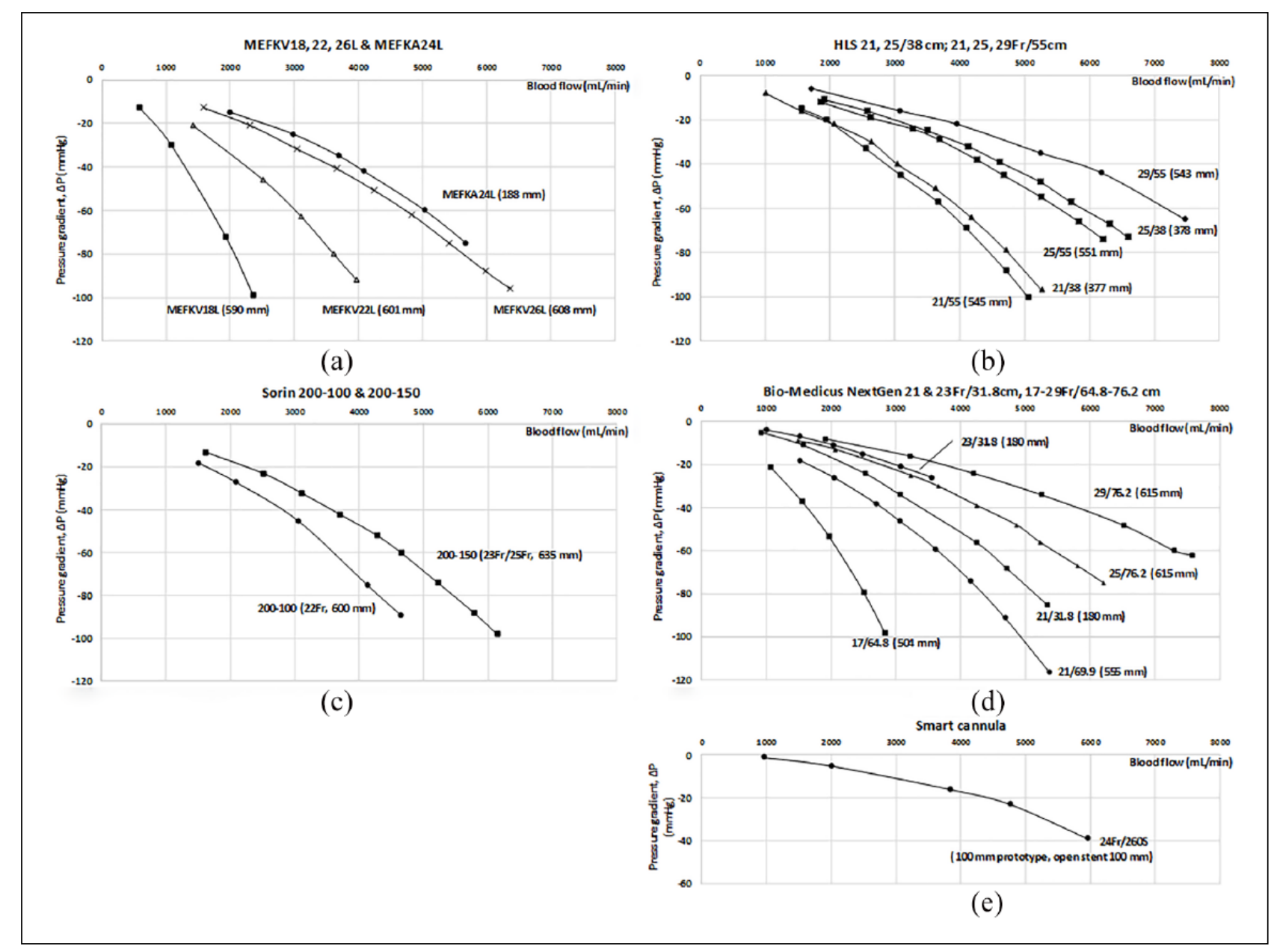

Figure 3. Pressure-flow relationship for different cannulae sizes from brand referred to in each panel. Cannula type and size/length according to manufacturer's preference shown at each bar in graph. Each panel head shows the manufacturers' nomenclature. The measured insertion length is presented in brackets next to the respective cannula curve (see also Table I). (a) the Xenios/Medos cannulae; (b) the Getinge/Maquet HLS cannulae; (c) the Sorin/Livanova cannulae; (d) the Medtronic/Bio-Medicus NextGen cannulae; and (e) the Eurosets Smart draining device (Smart cannula). Fr = French, I Fr= I/3 mm (outer cannula diameter).

ECMO systems are pump driven (either roller or centrifugal). Both types of pump require low resistance to flow and adequate preload or pre-priming for optimal performance. ${ }^{15}$ Centrifugal pumps create significant negative pressures to ensure blood flow. ${ }^{16}$ Higher shear rates and increased negative pressures enhance the risk of cavitation and formation of microbubbles in the pump, ${ }^{17}$ as well as the risk of hemolysis possibly resulting in increased inflammation and coagulation activation, in turn leading to adverse events. ${ }^{16}$ The experimental data clearly demonstrated that volume flow was higher for given $\Delta \mathrm{P}$ in larger diameter cannulae, and that larger diameter cannulae needed a lower $\Delta \mathrm{P}$ for a given flow. This is due to that the friction losses are inversely proportional to the cannula diameter to the power of five. ${ }^{18}$ Thus, a decrease in diameter is associated with an increase in friction losses, requiring a larger pressure gradient to provide the same blood flow in the smaller cannula. It therefore seems reasonable to hypothesize that shear stress-related complications for a given flow rate may decrease if combining a centrifugal pump and larger diameter drainage cannula. Moreover, the level of shear stress developing in the flow is directly associated with the flow being considered laminar, transitional, or turbulent. Each cannula would have an "optimal flow range." In this range, blood flow is likely to be laminar with low dynamic viscosity due to shear thinning, and thus exert minimal mechanical stress to blood components. The flow is below the zone where it starts to transition into turbulence but still high enough to keep from flow stagnation. However, to evaluate this was not the aim of this study.

Cannula tips for peripheral cannulae, except for the Smart cannula (blunt), carried at least six side holes in addition to the primary end hole. The blunt tip cannulae design has in an earlier study been shown to result in the highest shear forces. ${ }^{19}$ In the current investigation, the 24Fr Smart cannula was the only device that 


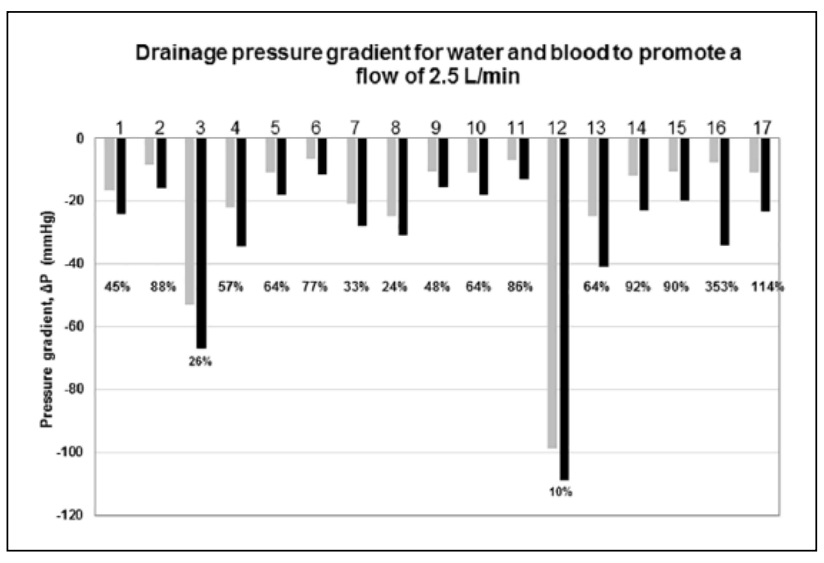

Figure 4. Figure 4 shows the net drainage pressure, $\Delta \mathrm{P}$, for water (gray) and for blood (hematocrit $27 \%$, black) to accomplish a flow of $2.5 \mathrm{~L} / \mathrm{min}$. The fractional (\%) additional pressure needed for blood compared to water is presented under each cannula. The cannulae are anonymized and arranged in order from (left to right) by decreasing diameter and increasing length. Individual design not considered and the data only assessed at one static flow. The water flow data were extracted from charts published by the manufacturers on Internet. The Smart Cannula was not included in the figure due to lack of data on water.

approximated a blunt tip. However, the stent surrounding this tip may act like a Cage tip which may alter the flow properties. This has, to our knowledge, not been investigated. The side holes are probably also important, not only for flow but also for blood component interaction and activation. ${ }^{19,20}$

Cannulae have been developed to allow ECMO configurations preferred by most clinicians to reduce recirculation fraction (for veno-venous (VV) ECMO) and to comply with the cannulator's preferences. The dominant access site for ECMO cannulation in adults are via the femoral veins, with the tip advanced to the upper inferior vena cava (IVC), right atrium (RA), or superior vena cava (SVC). These cannulae often have a second circumferential segment of draining holes approximately $200 \mathrm{~mm}$ downstream from the tip (Table 1 ). The multi-staged continuous or multi-stage interrupted cannulae drain preferentially from the most proximal holes (furthest from the tip) unless venous obstruction prevents this from occurring. ${ }^{21}$ To reduce the recirculation fraction on VV ECMO, the cannula tips need to be physically separated. ${ }^{22,23}$ For veno-arterial (VA) ECMO, it is preferable to drain from both the SVC and RA to maximize access to deoxygenated blood and to reduce the impact of the differential hypoxemia of the upper and lower body. ${ }^{24,25}$ Both of these circumstances require a long drainage cannula when placed via a femoral vein. The data from the current study reinforce the implication of Poiseuille's law, that to maximize volume flow, the drainage cannula should also be of a larger diameter. Unfortunately, the larger the diameter, the greater the risk of cannula-related complications including venous obstruction, leg edema, and deep venous thrombosis. ${ }^{26,27} \mathrm{~A}$ thinner shorter cannula may reduce some of the adverse events associated with larger diameter cannula as well as reducing thrombogenicity and inflammation due to exposure of blood to larger artificial surface and, for example, presence of mid-cannula drainage holes. To exemplify, in Figure 3(a), the Medos MEFKA24L performed slightly better than the longer MEFKV26L, and in Figure 3(d), the Medtronic 23/31.8 behaved similar to the multi-staged interrupted Medtronic 25/76.2 cannula. It may thus be tempting to sort out long and short cannulae of different diameters into groups of "sweet zones" of similar estimated flows for guidance of cannula choice. However, to determine optimal cannula from only diameter, insertion length and pressure-flow curves are not sufficient. The aspect of blood trauma also needs to be considered. The determination of a cannula's optimal flow range requires investigations at different hematocrits of flow speed, friction losses, and shear rates for each cannula (dimension and design). For the clinician though, maximum applied suction, that is, negative pressure applied proximal to the cannula for drainage, estimated required flow, cannulation configuration to be provided, and hematocrit, would be the variables to decide from.

Devices such as cannulae are regulated and controlled according to different standards (International Standard, ISO 10993-4, ISO 13485) and as for the European Union (EU) directives Medical Devices Directives (MDD). A new EU regulation, the Medical Devices Regulation (MDR EU 2017/745), will be fully implemented during 2020. ${ }^{28}$ The regulations and standards allow testing of flow characteristics using water, hence manufacturers are fully compliant with the regulations. The results of this experimental study demonstrate that there is a significant difference in performance between testing with water and HWB and that, to better inform clinicians about the characteristics of cannulae (and other devices used in ECMO), testing should provide data using blood of a standardized hematocrit.

A limitation of this study was that cannulae could not be compared due to specific request from industry to provide test cannulae. The fact that no tests were performed with water for replication of manufacturer's published data would also be regarded as a limitation. Blood with a constant hematocrit of $27 \%$ was used. The hematocrit target does vary between institutions and also often on an individual patient basis with some centers accepting lower values and others aiming at higher values than $27 \% .^{13,14}$ However, we were limited by the fact that all HWB available was delivered and used for priming of the mock loop. It is known that the effect of shear thinning is different for different hematocrits and shear rates..$^{29}$ Our experimental data clearly demonstrate 
a shear thinning effect. However, although it is known that shear thinning varies with changes in hematocrit, its effect on cannula performance in vivo remains unknown. Additional limitations are that it is possible that the flow characteristics of HWB which is over 42 days is different to fresh blood in vivo. Similarly, as HWB was used, it is not possible to make a recommendation as to whether fresh animal blood will provide adequate information for clinicians.

\section{Conclusion}

The pressure-flow properties of a variety of drainage cannulae currently available for peripheral ECMO were independently investigated using HWB in an in vitro model. Independent of cannula, the flow was lower at any given pressure gradient pumping blood when compared with the charts presented by the manufacturers (usually using water). However, there is no uniform linear additional pressure to apply that could serve as a guide for the ECMO provider assuring that the patient's "needs" are met. The information provided by manufacturers does not correspond to clinical performance and therefore may not provide the best guidance for clinicians.

\section{Declaration of Conflicting Interests}

The author(s) declared the following potential conflicts of interest with respect to the research, authorship, and/or publication of this article: LMB, FST, MVM, MDN, FP, and MB are on the Medical Advisory Board of Eurosets Srl., Medolla, Modena, Italy. No conflicts of interest were reported for any of the other authors.

\section{Funding}

The author(s) received no financial support for the research, authorship, and/or publication of this article.

\section{Acknowledgements}

We are grateful to the manufacturers that supported this survey (alphabetical order): Eurosets Srl., Medolla, Modena, Italy; Getinge, Maquet Cardiopulmonary GmbH, Rastatt, Germany; Medtronic International Trading Sarl, Tolochenaz, Switzerland; Octopus Medical AB/Limedic AB, Täby, Sweden; Sorin/ Livanova, Milan, Italy; and Xenios AG, Heilbronn, Germany. LMB designed the concept, the test chamber, and mock loop; collected, compiled, and analyzed the data; and drafted the manuscript. RL co-designed the concept. CJW, MG, LPdC, and JS collected the data. JW constructed the test chamber. LMB, RL, CJW, MG, LPdC, JS, JW, LPW, FST, MVM, MDN, LV, NAB, $\mathrm{FP}, \mathrm{JB}, \mathrm{TM}$, and $\mathrm{MB}$ interpreted the data and critically revised the manuscript. Approval for submission: All authors.

\section{ORCID iDs}

LarsMikaelBroman (iD https://orcid.org/0000-0003-4124-4581 LisaPrahlWittberg iD https://orcid.org/0000-0001-9976-8316
Matteo Di Nardo (iD https://orcid.org/0000-0003-0051-8080 Justyna Swol iD https://orcid.org/0000-0002-2903-092X

Roberto Lorusso (iD) https://orcid.org/0000-0002-1777-2045

\section{References}

1. Brogan TV, Lequier L, Lorusso R, et al. (eds). Extracorporeal life support: the ELSO red book. 5th ed. Ann Arbor, MI: Extracorporeal Life Support Organization, 2017.

2. ELSO Registry Report. Extracorporeal life support organization (ELSO), https://www.elso.org/Registry/Statistics .aspx

3. Bartlett RH. ECMO: the next ten years. Egypt J Crit Care Med 2016; 4: 7-10.

4. Makdisi G, Wang IW. Extra corporeal membrane oxygenation (ECMO) review of a lifesaving technology. $J$ Thorac Dis 2015; 7: e166-e176.

5. Paulsen MJ, Orizondo R, Le D, et al. A simple, standard method to characterize pressure/flow performance of vascular access cannulas. ASAIO J 2013; 58: 24-29.

6. Tazraei P, Riasi A, Takabi B. The influence of the nonNewtonian properties of blood on blood-hammer through the posterior cerebral artery. Math Biosci 2015; 264: 119-127.

7. Anand M, Rajagopal KR. A shear-thinning viscoelastic fluid model for describing the flow of blood. Int $J$ Cardiovasc Med Sci 2004; 4: 59-68.

8. Chen S. Shear dependence of effective cell volume as a determinant of blood viscosity. Science 1970; 168: 977979.

9. Kohler K, Valchanov K, Nias G, et al. ECMO cannula review. Perfusion 2013; 28: 114-124.

10. Pries AR, Neuhaus D, Gaehtgens P. Blood viscosity in tube flow: dependence on diameter and hematocrit. Am J Physiol 1992; 236: H1770-H1778.

11. Wells RE, Merrill EW, Gabelnick H. Shear-rate dependence of viscosity of blood: interaction of red cells and plasma proteins. Trans Soc Rheol 1962; 7: 9-24.

12. Fraser KH, Taskin ME, Griffith BP, et al. The use of computational fluid dynamics in the development of ventricular assist devices. Med Eng Phys 2011; 33: 263-280.

13. Agerstrand CL, Burkart KM, Abrams DC, et al. Blood conservation in extracorporeal membrane oxygenation for acute respiratory distress syndrome. Ann Thorac Surg 2015; 99: 590-595.

14. Voelker MT, Busch T, Bercker S, et al. Restrictive transfusion practice during extracorporeal membrane oxygenation therapy for severe acute respiratory distress syndrome. Artif Organs 2015; 39: 374-378.

15. Veres JP (ed.). Centrifugal and axial pump design and off-design performance prediction (NASA Technical Memorandum 106745). Sunnyvale, CA: Propulsion Committee, 1994, https://ntrs.nasa.gov/archive/nasa/ casi.ntrs.nasa.gov/19950013379.pdf

16. Pedersen TH, Videm V, Svennevig JL, et al. Extracorporeal membrane oxygenation using a centrifugal pump and servo regulator to prevent negative inlet pressure. Ann Thorac Surg 1997; 63: 1333-1339.

17. Lin Z, Ruan X, Zou J, et al. Experimental study of cavitation phenomenon in a centrifugal blood pump induced 
by the failure of inlet cannula. Chin J Mech Eng 2014; 27: 165-170.

18. Fox RW, McDonald AT, Pritchard PJ, et al. (eds). Fluid mechanics. 9th ed. Hoboken, NJ: John Wiley \& Sons Inc., 2016, pp. 299-300.

19. Park JY. The need of slanted side holes for venous cannulae. Comput Math Methods Med 2012; 2012: 854938.

20. Fuchs G, Berg N, Broman LM, et al. Flow-induced platelet activation in components of the extracorporeal membrane oxygenation circuit. Sci Rep 2018; 8: 13985.

21. Lindholm JA. Cannulation for veno-venous extracorporeal membrane oxygenation. J Thorac Dis 2018; 10(Suppl. 5): S606-S612.

22. Abrams D, Bacchetta M, Brodie D. Recirculation in venovenous extracorporeal membrane oxygenation. ASAIO J 2015; 61: 115-121.

23. Palmér O, Palmér K, Hultman J, et al. Cannula design and recirculation during venovenous extracorporeal membrane oxygenation. ASAIO J 2016; 62: 737-742.

24. Hou X, Yang X, Du Z, et al. Superior vena cava drainage improves upper body oxygenation during veno-arterial extracorporeal membrane oxygenation in sheep. Crit Care 2015; 19: 68.
25. Lindfors M, Fernckner B, Sartipy U, et al. Venous cannula positioning in arterial deoxygenation during venoarterial extracorporeal membrane oxygenation-a simulation study and case report. Artif Organs 2017; 41: 75-81.

26. Menaker J, Tanbatabai A, Rector R, et al. Incidence of cannula-associated deep vein thrombosis after venovenous extracorporeal membrane oxygenation. ASAIO J 2017; 63: 588-591.

27. Cooper E, Burns J, Retter A, et al. Prevalence of venous thrombosis following venovenous extracorporeal membrane oxygenation in patients with severe respiratory failure. Crit Care Med 2015; 43: e581-e584.

28. Di Nardo M, Vercaemst L, Swol J, et al. A narrative review of the technical standards for extracorporeal life support devices (pumps and oxygenators) in Europe. Perfusion 2018; 33: 553-561.

29. Flormann D, Schirra K, Podgorski T, et al. On the rheology of red blood cell suspensions with different amounts of dextran: separating the effect of aggregation and increase in viscosity of the suspending phase. Rheologica Acta 2016; 55: 477-483. 